Sharif University of Technology
Scientia Iranica
SCIENTIA
IRAN I CA
http://scientiairanica.sharif.edu

\title{
Optimal objective function for simulating endurance time excitations
}

\author{
M. Mashayekhi*, H.E. Estekanchi, and H. Vafai \\ Department of Civil Engineering, Sharif University of Technology, Tehran, P.O. Box 11155-9313, Iran. \\ Received 16 October 2017; received in revised form 22 April 2018; accepted 10 November 2018
}

\author{
KEYWORDS \\ Endurance time \\ method; \\ Optimization; \\ Objective function; \\ Response spectra; \\ Time history dynamic \\ analysis.
}

\begin{abstract}
Endurance Time (ET) method is a dynamic analysis procedure in which increasing excitations are imposed on structures; these excitations are known as Endurance Time Excitation Functions (ETEF). This study presents a method to determine an optimal objective function for simulating ETEFs including unconstrained optimization problems. In optimization problems, equations are defined in terms of an objective function. In the problem of simulating ETEFs, the objective function can be defined in many different ways regarding the considered intensity measures and respective weighting factors. In addition, the type of calculating residuals (absolute way or relative way) diversifies the objective function definitions. The proposed method for determining the optimal objective function includes quantifying the accuracy of ETEFs in a scalar quantity regardless of their objective functions and introducing an approach to attenuate the dependence of results on the initial points of optimizations. The proposed method is applied, and results are then presented. It is observed that considering only acceleration spectra and calculating residuals in a relative way lead to more accurate ETEFs.

(C) 2020 Sharif University of Technology. All rights reserved.
\end{abstract}

\section{Introduction}

Endurance Time (ET) method is a dynamic analysis in which structures are subjected to intensifying acceleration time histories [1]. The ET method provides seismic demand prediction in terms of the correlation between Engineering Demand Parameter (EDP) and Intensity Measure (IM). EDP describes structural responses such as inter-story drift ratio, while IM represents seismic intensity levels such as acceleration spectra in the first structural mode. The ET method illustrates the performance of a structure at different seismic intensity levels.

\footnotetext{
*. Corresponding author.

E-mail addresses:

mohammad.mashayekhi67@student.sharif.edu (M.

Mashayekhi); stkanchi@sharif.edu (H.E. Estekanchi); vafai@sharif.edu (H. Vafai)
}

doi: $10.24200 /$ sci.2018.5388.1244
Several other procedures can be employed for the EDP prediction, e.g., Incremental Dynamic Analysis (IDA) and Nonlinear Static Procedure (NSP). NSP is a static procedure that does not consider the dynamic nature of building responses during earthquakes [2]. NSP is a simple approach presented by several rehabilitation provisions such as FEMA 356 [3] and FEMA273 [4]. In contrast, IDA is a time history dynamic-based procedure for the seismic assessment that subjects structures to a suite of multiple-scaled Ground Motion (GM) records [5]. Each scale factor creates a specific seismic intensity level. Thus, IDA gives an insight into the structural performance at different intensity levels, ranging from frequent events to rare earthquakes.

The ET method, as an EDP prediction tool, lies between NSP and IDA in terms of its simplicity and accuracy. This method is more accurate than NSP as a result of considering the dynamic nature of building responses. In addition, the ET method is simpler than the IDA analysis. In contrast to the IDA analysis 
that requires a set of dynamic analyses for each seismic intensity level, the ET method expresses overall structural performance using a single time-history analysis. Therefore, the ET method shortens the computational time of analysis. In addition, employing a suite of GM records for all intensity levels in the IDA analysis is a controversial issue [6].

The ET method is used in different areas of earthquake engineering such as seismic assessment, performance-based design, probabilistic-based earthquake engineering, etc. Riahi et al. [7] investigated the potentials and limits of the ET method for performing nonlinear seismic analysis of Single Degree Of Freedom (SDOF) structures. Riahi et al. [8] applied the ET method for performing the seismic assessment of steel frames with different stories and different bays. Mirzaee and Estekanchi [9] developed an ET-based methodology for performance-based retrofitting of typical steel frames. Rahimi and Estekanchi [10] applied the ET method to the collapse assessment of buildings. Basim and Estekanchi [11] investigated the application of the ET method to the performance-based design of structures and proposed a practical optimum design procedure. Tafakori et al. [12] presented an ET-based methodology to estimate probabilistic loss for which the ET method was used as a demand propagation prediction tool. Chiniforush et al. [13] applied the ET method for performing the seismic evaluation of unreinforced masonry monuments. Vaezi et al. [14] investigated the seismic response of anchored cylindrical steel tanks with various dimensional parameters considering fluid-structure interaction by the ET method.

The ET Excitation Functions (ETEF), which are used as time-history analysis input motions, are the central part of the ET method. ETEFs represent synthetic records and are generated mathematically. Dynamic characteristics of the ETEFs are supposed to be compatible with recorded GMs and also increase with time. Given that ETEF intensity increases in time, a wide range of IMs are covered in a single ETEF. Intensification is a key feature of ETEFs. Besides, the EDP calculated by the ET method must be compatible with the EDP obtained from the IDA analysis at each IM.

These two foregoing requirements include the simulation basis of ETEFs. ETEF-generating equations are defined as discrepancies between ETEFs dynamic characteristics and GMs. Since the variable numbers are quite smaller than the number of equations, the optimization procedure must be used to minimize equations. In the optimization context, equations are presented by objective functions.

There are several options when it comes to defining the objective function. Different objective function definitions vary in considered dynamic characteristics, residual calculation type, and residual weighting associated with different dynamic characteristics. Residuals are the differences between the ETEF dynamic characteristics and corresponding GMs. There are two types of residual calculation, i.e., absolute calculation and relative calculation.

Several studies have intended to simulate ETEFs, neither of which has focused on investigating the influence of objective function definition on the results. For example, Nozari and Estekanchi [15] applied an optimization procedure for generating ETEFs. In their study, residuals of absolute acceleration spectra were considered in the objective function. Kaveh and Mahdavi [16] presented a process for generating ETEFs based on discrete wavelet transform, which solely included acceleration spectra consistency. In their work, residuals were computed in an absolute manner. Heuristic optimization algorithms were also adopted by Kaveh et al. [17] for generating ETEFs. To simulate new ETEFs, it is essential to define an optimal objective function that creates better ETEFs.

Several studies have investigated the dynamic parameters so that they can be included in the ET method so as to either simulate ETEFs or apply the ET method. For example, Mashayekhi and Estekanchi [18] investigated the effect of strong motion duration in the ET analysis. Mashayekhi and Estekanchi [19] considered nonlinear cycle consistency of ETEFs with GMs in the existing ETEFs. Mashayekhi and Estekanchi [20] also investigated the number of cycles in which ETEFs show consistency with GMs.

The problem of simulating ETEFs relies heavily on optimization. Diverse optimization algorithms have been introduced in the literature; these algorithms have been widely used to solve many optimization problems in various scientific fields. In the problem of simulating ETEFs, several studies have applied different optimization algorithms. The performance of those algorithms has been compared with each other. Nozari and Estekanchi [15] applied Trust-Region reflective method to simulate ETEFs. Kaveh and Mahdavi [16] and Kaveh et al. [17] applied nonlinear Quasi-Newton algorithm and Covariance Matrix Adaption (CMA) evolutionary strategy approach to generate ETEFs, respectively. It was shown that the Trust-Region reflective method could produce better results than other considered algorithms. It is worth mentioning that even though those algorithms are characterized by some advantages that speed up their convergence, they cannot compete with the accuracy of the trustregion reflective algorithm. Since accuracy is the most important issue in the problem of simulating ETEFs, Trust-Region reflective algorithm is used in this study.

This study focuses on simulating new ETEFs. Although optimization algorithm affects results, this study concentrates only on the objective function definition. A novel method is proposed to find the optimal objective function definition. The method is employed 
to find the optimal ETEFs simulation scenario, where acceleration, displacement, and velocity spectra are included in the process. The accuracy of ETEFs simulated by the optimal objective function is compared with that of ETEFs simulated by conventional objective functions.

\section{Generating procedure}

The concept of Response Spectra is used in ETEFs generation. It is expected that ETEFs response spectra increase with time whilst they remain compatible with the recorded GMs. This requirement for acceleration spectra is shown in Eq. (1):

$$
S_{a C}(t, T)=t / t_{\text {target }} * S_{a}^{\text {TARGET }}(T),
$$

where $S_{a C}(t, T)$ denotes the acceleration spectra produced by window $[0, t]$ of ETEFs in time period T. $S_{a}^{\text {TARGET }}(T)$ denotes the average recorded GMs acceleration spectra. In this study, FEMAP695 [21] far-field record set is used. These GMs acceleration spectra are shown in Figure 1.

The ETEFs requirement for displacement and velocity spectra are shown in Eqs. (2) and (3), respectively:

$$
\begin{aligned}
& S_{u C}(t, T)=t / t_{\text {target }} * S_{u}^{\text {TARGET }}(T), \\
& S_{v C}(t, T)=t / t_{\text {target }} * S_{v}^{\text {TARGET }}(T),
\end{aligned}
$$

where $S_{u C}(t, T)$ is the target displacement spectra pro-

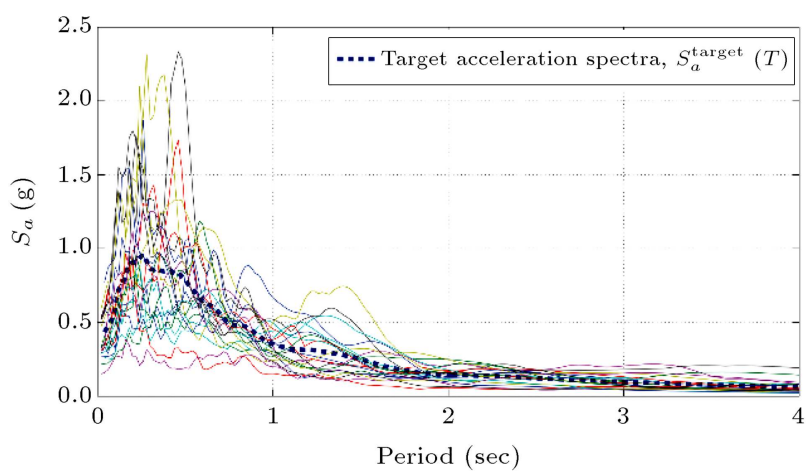

Figure 1. Acceleration spectra of FEMAp695 record set. duced by window $[0, t]$ of ETEFs at time $t$ and in period $T, S_{d}^{\mathrm{TARGET}}(T)$ represents the average recorded GMs displacement response spectra in period $T, S_{v c}(t, T)$ is the velocity spectra produced by the ETEF at time $t$ and in period $T$, and $S_{v}^{\text {TARGET }}(T)$ is recorded GMs average velocity spectra in period $T$.

The ETEFs response spectra must conform to Eqs. (1), (2), and (3). These equations are the ETEFs simulating targets. The ETEFs response spectra are evaluated through Eqs. (4a), (4b), and (4c):

$$
\begin{aligned}
& S_{a}(t, T)=\max \left(\left|\ddot{x}(\tau)+a_{g}(\tau)\right|\right) \quad 0 \leq \tau \leq t, \\
& S_{u}(t, T)=\max (|x(\tau)|) \quad 0 \leq \tau \leq t, \\
& S_{v}(t, T)=\max (|\dot{x}(\tau)|) \quad 0 \leq \tau \leq t,
\end{aligned}
$$

where $\ddot{x}(\tau), x(\tau)$, and $\dot{x}(\tau)$ are the relative accelerations, displacement, and velocity response of a SDOF system in period $T$ and at a damping ratio of $5 \%$ under the ETEF, and $a_{g}(\tau)$ is the acceleration time history of the ETEF.

To solve the above-mentioned equations, unconstrained nonlinear optimization is utilized. The objective function of this optimization problem is defined as in Eq. (5) shown in Box I. It should be noted that this objective function intends to minimize the residuals defined as the differences between the ETEFs response spectra and targets. This objective function integrates absolute residuals over all times and all periods. The first three terms in the objective function compute residuals in an absolute manner, while the other three terms compute residuals in a relative manner. In Eq. 2(5) $t_{\max }$ is the ETEF duration, and $T_{\max }$ is the maximum period considered in generating. $\boldsymbol{\alpha}$ is the weight vector, that is $\boldsymbol{\alpha}=$

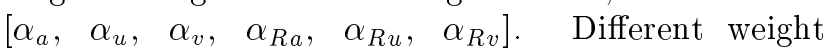
vectors produce different optimization scenarios. $\boldsymbol{\alpha}$ determines residual weight factors of each component in the objective function. For example, when all components of the weight vector are zero except $\alpha_{a}$, it can be found that residuals of absolute acceleration spectra are considered in the objective function. In this study, optimization scenarios are defined based on the values of weight vectors. $\alpha_{a}$ is the factor of

$$
\begin{aligned}
F_{\mathrm{ETEF}}\left(a_{g}, \alpha\right)= & \int_{0}^{T_{\max }} \int_{0}^{t_{\max }}\left\{\alpha_{a}\left[S_{a}(T, t)-S_{a C}(T, t)\right]^{2}+\alpha_{u}\left[S_{u}(T, t)-S_{u C}(T, t)\right]^{2}+\alpha_{v}\left[S_{v}(T, t)-S_{v C}(T, t)\right]^{2}\right. \\
& \left.+\alpha_{R a}\left[\frac{S_{a}(T, t)-S_{a C}(T, t)}{S_{a C}(T, t)}\right]^{2}+\alpha_{R u}\left[\frac{S_{u}(T, t)-S_{u C}(T, t)}{S_{u C}(T, t)}\right]^{2}+\alpha_{R v}\left[\frac{S_{v}(T, t)-S_{v C}(T, t)}{S_{v C}(T, t)}\right]^{2}\right\} d t d T .
\end{aligned}
$$


acceleration spectra residuals and is computed in an absolute manner. This factor is always valued at one. $\alpha_{R a}, \alpha_{R u}, \alpha_{R v}$ represent the factors of acceleration, displacement, and velocity spectra computed in a relative manner and are either zero or one. This implies that the relative error of these quantities is either considered or not. It should be mentioned that velocity spectra consistency is not considered, solely because it is less important than displacement spectra. $\alpha_{u}, \alpha_{v}$ are the displacement and residuals of velocity spectra, which are computed in an absolute manner. These factors are assigned either zero or one in the literature. This study introduces a new method for assigning these factors in which the importance of displacement spectra and velocity spectra residuals in the objective function is the same. These factors are measured according to the following equations:

$$
\begin{gathered}
\alpha_{u}=\frac{\int_{T_{\min }}^{T_{\max }} S_{a}^{\text {TARGET }}(T) \mathrm{d} T}{\int_{T_{\min }}^{T_{\max }} S_{u}^{\text {TARGET }}(T) \mathrm{d} T}, \\
\alpha_{v}=\frac{\int_{T_{\min }}^{T_{\max }} S_{a}^{\text {TARGET }}(T) \mathrm{d} T}{\int_{T_{\min }}^{\text {TAx }} S_{v}^{\text {TARGET }}(T) \mathrm{d} T} .
\end{gathered}
$$

It should be noted that the above-mentioned objective functions should be discretized so that they can be minimized. The discretization type can impact the results. Suppose that time is sampled at $n$ points $\left(t_{j}, j=1: n\right)$ and periods are sampled at $m$ points $\left(T_{i}, i=1: m\right)$; then, this discretization converts the double integral into a double summation. The objective function in Eq. (5) changes into Eq. (8) as shown in Box II, after discretization. In this study, the aim is to generate 20-sec acceleration time histories of ETEFs that are sampled at a time step of $0.01 \mathrm{sec}$. Thus, this sampling method defines 2000 variables, which must be specified during the optimization process. Period $T$ is discretized at 120 points with logarithmic distribution at interval $[0.02 \mathrm{sec}, 5 \mathrm{sec}]$. The logarithmic distribution generates more data points in the lower period region, where the fluctuation of acceleration spectra is considerably high.

Nonlinear unconstrained optimization was used to determine the variables. This study used the trustregion reflective method as an optimization algorithm, which is a simple yet powerful concept in the field of optimization. The basic idea is to approximate $f$ with a simpler function $q$, which reasonably reflects the behavior of function $f$ in the neighborhood of $N-$ a spherical space with a radius of $\Delta$-around the point $x$. This neighborhood is called the trust region [22]. In the standard trust region, $q$ is defined by the first two terms in the Taylor expansion of $f$ around $x$. The basic equation of the trust region method is a constrained optimization problem, which is given in Eq. (9) [22]:

$$
\operatorname{minimize}\left\{\frac{1}{2} s^{T} H s+s^{T} g\right\} \text { such that }\|s\| \leq \Delta .
$$

The gradient of $f$ at $x$ is represented by $g$, while $H$ is the Hessian matrix (the symmetric matrix of the second derivatives) of $f$ at $x$, and $s$ is the step size to be determined in each iteration. When $s$ is determined, the point of the next iteration is calculated by adding the step size to the current point. This procedure is repeated until convergence is reached. The ETEFgenerating algorithm is depicted in Figure 2. It can be seen that the optimization run terminates as soon as one of the convergence criteria is satisfied. The convergence criteria used in this study include the following:

- Iteration number reaches a specified value. In this study, maximum iteration number 200 is considered;

- The number of function evaluations reaches a specified value. In this study, the maximum number of function evaluations is considered 400000;

$$
\begin{aligned}
F_{\mathrm{ETEF}}\left(a_{g}, \alpha\right)= & \sum_{i=1}^{m} \sum_{j=1}^{n}\left\{\alpha_{a}\left[S_{a}\left(T_{i}, t_{j}\right)-S_{a C}\left(T_{i}, t_{j}\right)\right]^{2}+\alpha_{u}\left[S_{u}\left(T_{i}, t_{j}\right)-S_{u C}\left(T_{i}, t_{j}\right)\right]^{2}\right. \\
& \left.+\alpha_{v}\left[S_{v}\left(T_{i}, t_{j}\right)-S_{v C}\left(T_{i}, t_{j}\right)\right]^{2}+\alpha_{R a}\left[\frac{S_{a}\left(T_{i}, t_{j}\right)-S_{a C}\left(T_{i}, t_{j}\right)}{S_{a C}\left(T_{i}, t_{j}\right)}\right]^{2}+\alpha_{R u}\left[\frac{S_{u}\left(T_{i}, t_{j}\right)-S_{u C}\left(T_{i}, t_{j}\right)}{S_{u C}\left(T_{i}, t_{j}\right)}\right]^{2}\right] \\
& \left.+\alpha_{R v}\left[\frac{S_{v}\left(T_{i}, t_{j}\right)-S_{v C}\left(T_{i}, t_{j}\right)}{S_{v C}\left(T_{i}, t_{j}\right)}\right]\right\}
\end{aligned}
$$




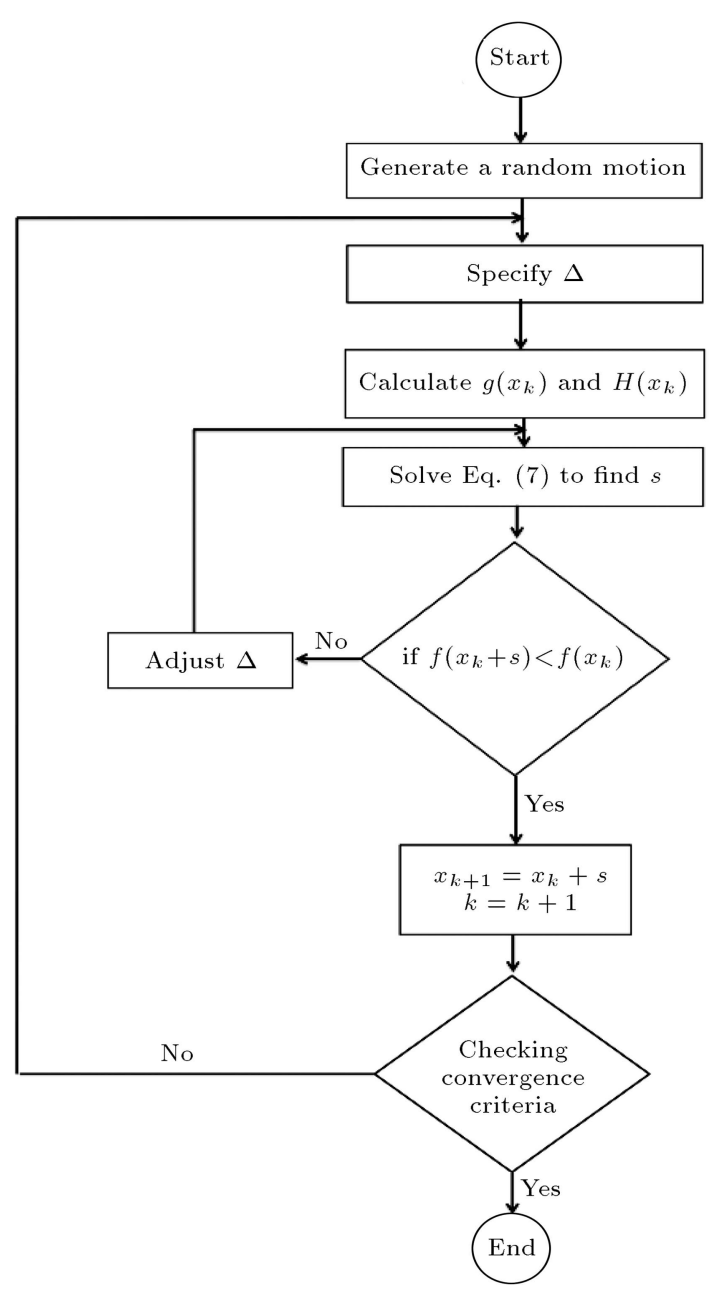

Figure 2. Generating Endurance Time Excitation Functions (ETEFs) algorithm.

- Size of the calculated step, which is the norm of $x_{k+1}-x_{k}$, is less than a specified value $\left(\left|x_{k}-x_{k+1}\right|<10^{-8}\left(1+\left|x_{k}\right|\right)\right)$;

- Changes in the objective function value during a step are less than a specified value. $\left|f(x)_{k}-f\left(x_{k+1}\right)\right|<10^{-8}$.

\section{Proposed method}

The proposed method is a procedure employed for finding the optimal ETEFs simulating objective function. The objective function parameters must be specified first.

Weight factor vector $(\boldsymbol{\alpha})$ is considered as the objective function parameter of ETEFs. Different weight factor values define different simulating scenarios. It is necessary to determine the best value for this parameter, which is of concern in generating new ETEFs.

In this regard, a criterion must be defined to compare the accuracy of ETEFs shown in different scenarios. In this section, the desired criterion is developed. However, the developed criteria might vary for different applications.

The Normalized Relative Residual (NRR) for each response spectra quantity is separately computed according to Eqs. (10), (11), (12):

$$
\begin{aligned}
N R R_{S_{a}}= & \frac{1}{t_{\max }} \int_{0}^{t_{\max }} \\
& \left(\frac{\int_{T_{\min }}^{T_{\max }}\left|\left(S_{a}(T, t)-S_{a C}(T, t)\right)\right| d T}{\int_{T_{\min }}^{T_{\max }} S_{a C}(T, t) d T}\right) d t, \\
N R R_{S_{u}}= & \left.\frac{1}{t_{\max }} \int_{0}^{t_{\max }}\right) d t \\
& \left(\frac{\int_{T_{\min }}^{T_{\max }}\left|\left(S_{u}(T, t)-S_{u C}(T, t)\right)\right| d T}{N_{T_{\min }}} S_{u C}(T, t) d T\right. \\
N_{S_{v}}= & \left.\frac{1}{t_{\max }} \int_{0}^{t_{\max }}\right)_{(12)}
\end{aligned}
$$

NRR integrates residuals in all time periods. The residuals in each time period are integrated in all periods and, then, are normalized. This normalization method avoids the domination of residuals, where the values of response spectra are lower and, thus, they can be divided by lower numbers. Total Relative Residual (TRR) is a vector, components of which are NRRs associated with $S_{a}, S_{u}$, and $S_{v}$. The concept of normalized residuals was first introduced by Mashayekhi et al. [23]. This quantity is a vector and, thus, cannot be used as a comparison tool. Total Relative Cost (TRC) is a comparison criterion that is derived from the inner product of TRR and importance vector $(\mathbf{I})$. Importance vector is a unit vector, components of which signify the accuracy importance of each response spectra quantity in simulated ETEFs. Schematic computation of TRC is shown in Figure 3. TRC is computed through Eq. (13):

$$
\mathrm{TRC}=\mathbf{T R R} . \mathbf{I}=\left[\mathrm{TRR}_{S_{a}}, \mathrm{TRR}_{S_{u}}, \mathrm{TRR}_{S_{v}}\right] . \mathrm{I},
$$

where "." denotes the inner product operator of two vectors.

The optimal $\boldsymbol{\alpha}$ can be specified by minimizing TRC. The following steps must be taken:

1. Consider a number of optimal possible scenarios; $n$ scenarios are numbered as $S C_{i}, i=1: n$ and each scenario has its own $\boldsymbol{\alpha}_{i}$. It should be noted that the optimal scenario is selected among these considered scenarios. Therefore, all possible scenarios should be considered. All possible scenarios have been discussed in the previous section; 


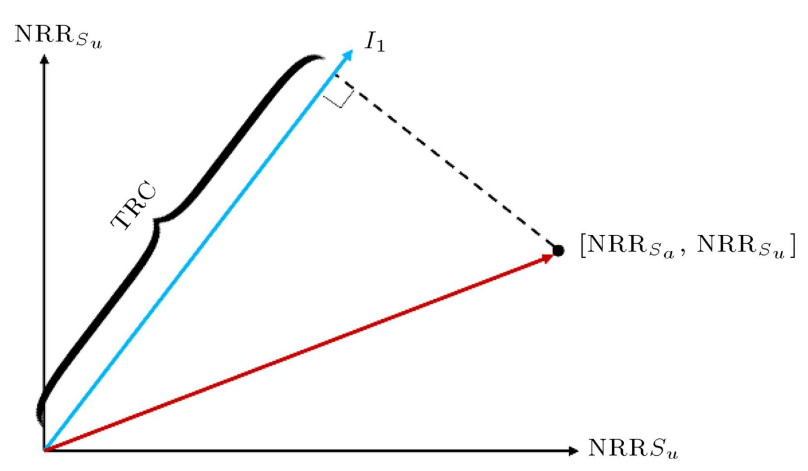

Figure 3. Computing total relative cost procedure.

2. Specify an importance vector;

3. Create a number of initial random solutions. $m$ initial random solution is numbered as $X_{j}, j=1$ : $m$;

4. Simulate $\operatorname{ETEFs}(i, j)$ using the $i$ th scenario and the $j$ th initial random solution. This step is done by optimizing Eq. (8);

5. Compute TRC matrix; each entry of this matrix is the TRC associated with a scenario and a random initial motion TRC $(i, j)$ associated with ETEFs $(i, j)$;

6. Normalize the TRC matrix; entries of each column are divided by the maximum entry of that column. In fact, the TRC of different scenarios associated with the same input motion is normalized with respect to each other;

7. Calculate the average TRC of each scenario by averaging rows of the normalized TRC matrix. In fact, the TRC of each scenario equals the average of the normalized TRC of that scenario with different random initial motions;

8. Sort different scenarios from the lowest to highest. The optimal scenario is the one with the minimum normalized TRC.

\section{Application}

In this section, the proposed method is applied to find the optimum $\boldsymbol{\alpha}$ vector. Nine scenarios are considered; the corresponding $\boldsymbol{\alpha}$ vectors are summarized in Table 1. For example, in the first scenario, the residual of absolute acceleration spectra is considered in the objective function, while, in the second scenario, the residual of relative acceleration spectra is considered in the objective function. In the third scenario, the summation of the residuals of absolute acceleration and displacement spectra is included in the objective function.

Three initial points are investigated. The first initial point $\left(X_{j}, j=1\right)$ is shown in Figure 4 . It should
Table 1. Characteristics of defined scenarios.

\begin{tabular}{cccc}
\hline Scenarios & {$\left[\begin{array}{llllll}1 & \alpha & 0 & 0 & 0 & 0\end{array}\right]$} \\
\hline SC1 2 & {$\left[\begin{array}{llllll}0 & 0 & 0 & 1 & 0 & 0\end{array}\right]$} \\
SC3 & {$\left[\begin{array}{llllll}1 & 1 & 0 & 0 & 0 & 0\end{array}\right]$} \\
SC4 & {$\left[\begin{array}{llllll}1 & 13 & 0 & 0 & 0 & 0\end{array}\right]$} \\
SC5 & {$\left[\begin{array}{llllll}0 & 0 & 0 & 1 & 1 & 0\end{array}\right]$} \\
SC6 & {$\left[\begin{array}{llllll}1 & 1 & 0 & 1 & 1 & 0\end{array}\right]$} \\
SC7 & {$\left[\begin{array}{llllll}1 & 1 & 1 & 0 & 0 & 0\end{array}\right]$} \\
SC8 & {$\left[\begin{array}{llllll}1 & 13 & 4 & 0 & 0 & 0\end{array}\right]$} \\
SC9 & {$\left[\begin{array}{llllll}1 & 13 & 4 & 1 & 1 & 1\end{array}\right]$} \\
\hline
\end{tabular}

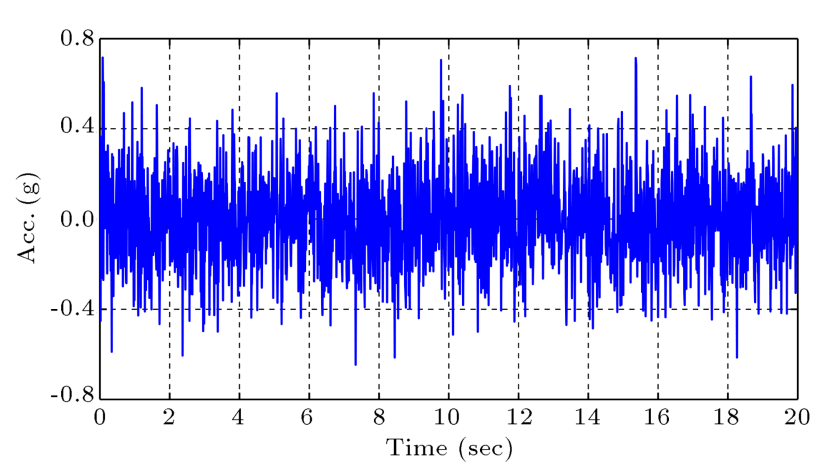

Figure 4. Initial point of optimization.

be mentioned that the units of acceleration, velocity, and displacement are g, g.sec, and g.sec ${ }^{2}$, respectively. The non-zero $\alpha_{u}$ and $\alpha_{v}$ values in the objective function are set to either 1 or the value computed by Eqs. (6) and $(7)$. These equations are used as normalization factors.

Two importance vectors are considered in order to investigate the sensitivity of the optimal scenario to this quantity, i.e., $\mathbf{I}_{1}=\left[\begin{array}{lll}\frac{1}{\sqrt{3}} & \frac{1}{\sqrt{3}} & \frac{1}{\sqrt{3}}\end{array}\right]$ and $\mathbf{I}_{2}=$ $\left[\begin{array}{lll}\frac{1}{\sqrt{2}} & \frac{1}{\sqrt{2}} & 0\end{array}\right] . \quad$ TRC associated with $\mathbf{I}_{1}$ and $\mathbf{I}_{2}$ is denoted by $\mathbf{T} \mathbf{R} \mathbf{C}_{\mathbf{1}}$ and $\mathbf{T} \mathbf{R} \mathbf{C}_{\mathbf{2}}$, respectively. Table 2 shows the NRR and TRC associated with these scenarios for the first initial point $\left(X_{1}\right)$.

It can be concluded that the fifth scenario that considers the relative residual of acceleration and the displacement spectra creates better $\mathbf{T} \mathbf{R} \mathbf{C}_{\mathbf{1}}$ and $\mathbf{T} \mathbf{R} \mathbf{C}_{\mathbf{2}}$. 
Table 2. NRR and TRC of optimizations scenarios.

\begin{tabular}{cccccc}
\hline Scenarios & TRR $_{\boldsymbol{S}_{\boldsymbol{a}}}$ & $\mathbf{T R R}_{\boldsymbol{S}_{\boldsymbol{d}}}$ & $\mathbf{T R R}_{\boldsymbol{S}_{\boldsymbol{v}}}$ & $\mathbf{T R C}_{\mathbf{1}}$ & $\mathbf{T R C}_{\mathbf{2}}$ \\
\hline SC1 & 0.14 & 0.16 & 0.18 & 0.277 & 0.210 \\
SC2 & 0.13 & 0.14 & 0.15 & 0.244 & 0.192 \\
SC3 & 0.15 & 0.16 & 0.19 & 0.288 & 0.219 \\
SC4 & 0.15 & 0.16 & 0.17 & 0.275 & 0.218 \\
SC5 & 0.12 & 0.14 & 0.15 & 0.239 & 0.185 \\
SC6 & 0.13 & 0.15 & 0.15 & 0.245 & 0.191 \\
SC7 & 0.17 & 0.19 & 0.21 & 0.324 & 0.251 \\
SC8 & 0.15 & 0.16 & 0.17 & 0.278 & 0.219 \\
SC9 & 0.13 & 0.15 & 0.14 & 0.242 & 0.198 \\
\hline
\end{tabular}

Table 3. Total relative cost for three different initial motions.

\begin{tabular}{|c|c|c|c|c|c|c|}
\hline \multirow{2}{*}{ Scenarios } & \multicolumn{2}{|c|}{$x_{1}^{(0)}$} & \multicolumn{2}{|c|}{$x_{2}^{(0)}$} & \multicolumn{2}{|c|}{$x_{3}^{(0)}$} \\
\hline & TRC $_{1}$ & $\mathrm{TRC}_{2}$ & TRC $_{1}$ & $\mathrm{TRC}_{2}$ & TRC $_{1}$ & $\mathrm{TRC}_{2}$ \\
\hline SC1 & 0.28 & 0.21 & 0.28 & 0.22 & 0.34 & 0.27 \\
\hline $\mathrm{SC} 2$ & 0.24 & 0.19 & 0.24 & 0.18 & 0.25 & 0.19 \\
\hline $\mathrm{SC} 3$ & 0.29 & 0.22 & 0.28 & 0.23 & 0.37 & 0.29 \\
\hline $\mathrm{SC} 4$ & 0.28 & 0.22 & 0.29 & 0.21 & 0.27 & 0.21 \\
\hline SC5 & 0.24 & 0.18 & 0.39 & 0.32 & 0.26 & 0.20 \\
\hline SC6 & 0.24 & 0.19 & 0.30 & 0.24 & 0.27 & 0.21 \\
\hline $\mathrm{SC} 7$ & 0.32 & 0.25 & 0.35 & 0.30 & 0.36 & 0.30 \\
\hline $\mathrm{SC} 8$ & 0.28 & 0.22 & 0.26 & 0.22 & 0.31 & 0.25 \\
\hline SC9 & 0.24 & 0.20 & 0.25 & 0.21 & 0.25 & 0.21 \\
\hline
\end{tabular}

This scenario creates minimum $\mathbf{T} \mathbf{R} \mathbf{C}_{\mathbf{1}}$ and $\mathbf{T} \mathbf{R} \mathbf{C}_{\mathbf{2}}$ in comparison to other scenarios. $\mathbf{T R C}_{\mathbf{1}}$ of $\mathrm{SC} 5$ is 0.239 and is better than other scenarios. It should be noted that although SC5 is the best scenario, the difference between TRC of SC9, SC2, and SC6 and that of SC5 is not significant. It is interesting that although $\mathrm{SC} 1$ and $\mathrm{SC} 2$ consider only acceleration spectra, the $\mathrm{TRR}_{S_{a}}$ associated with $\mathrm{SC} 5$ is better than $\mathrm{SC} 1$ and $\mathrm{SC} 2$.

$\mathbf{T R C}_{\mathbf{1}}$ and $\mathbf{T} \mathbf{R C}_{\mathbf{2}}$ results associated with three initial points are reported in Table 3 . Unlike the first motion in which the fifth scenario produced the best results, in the second and third motions, the second scenario is the best one. Therefore, it can be concluded that the best scenario is dependent on the initial motion.

In order to find the best scenario regardless of the effect of initial points, average TRC is computed in line with Step 7 of the proposed method. Figure 5 shows the average TRC of different scenarios. The simulation scenarios are sorted based on average $\mathbf{T R C}_{\mathbf{1}}$ and $\mathbf{T} \mathbf{R} \mathbf{C}_{\mathbf{2}}$. The sorted results are shown in Figure 6.

It is shown that different scenarios are sorted based on average $\mathrm{TRC}_{1}$ and $\mathrm{TRC}_{2}$. The sorted scenarios are shown in Figure 6. It can be seen that the order of scenarios based on $\mathrm{TRC}_{1}$ and $\mathrm{TRC}_{2}$ is identical. Although the importance vector is necessary to quantify ETEFs accuracy, it does not affect the

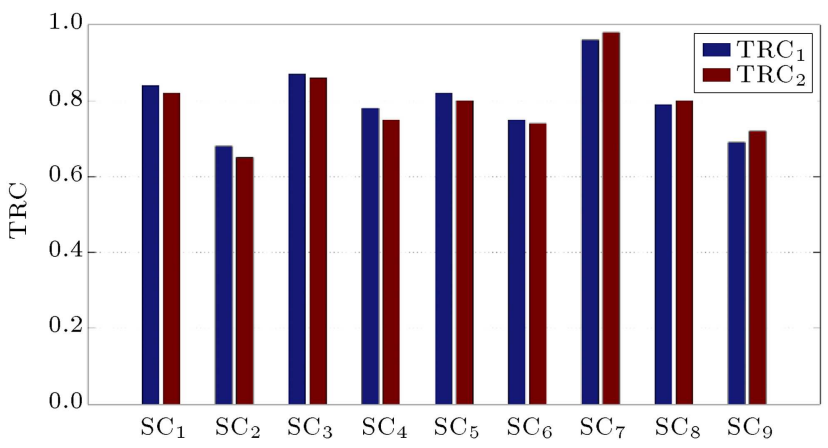

Figure 5. Total relative cost of different optimization scenarios.

optimal scenario and the order of scenarios from the lowest to highest. This can be justified based on the finding that the information concerning the acceleration, displacement, and velocity spectra is considered similar and that each can be converted into another by multiplication by angular frequency, as shown in Eq. (14):

$$
S_{u}(T)=S_{a}(T) \times\left(\frac{T}{2 \pi}\right)^{2},
$$

where $S_{u}(T)$ and $S_{a}(T)$ are displacement and acceleration spectra of motions in structural period $T$, respectively. 


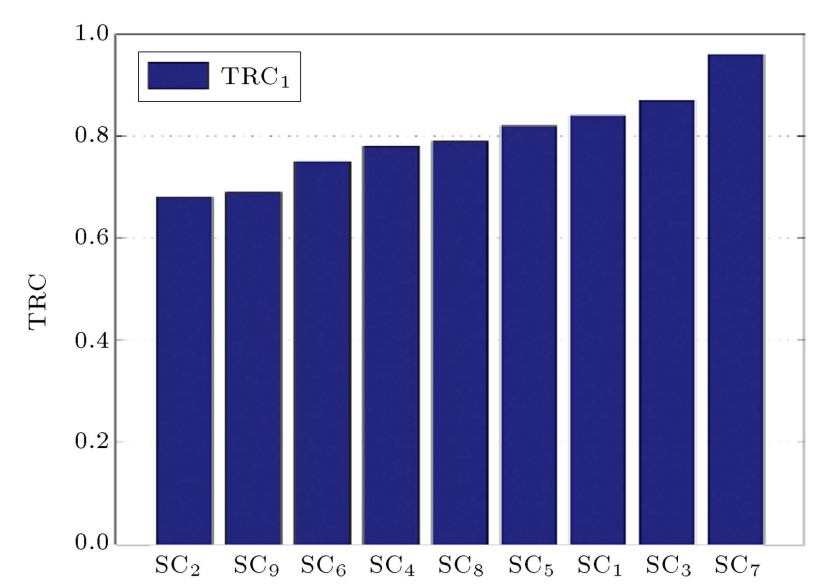

(a)

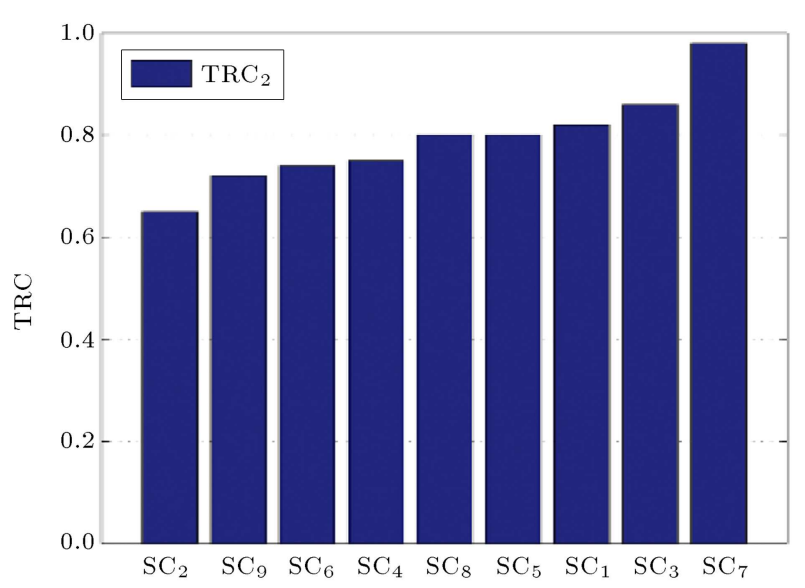

(b)

Figure 6. Sort of different simulation scenarios based on (a) $\mathrm{TRC}_{1}$ and (b) $\mathrm{TRC}_{2}$.

Because the ETEF-generating process is an optimization-based procedure, the objective function definition influences the process of finding optimum directions towards the best solution.

It can be seen that the second scenario is the optimal objective function definition. The residuals of relative acceleration spectra in the objective function result in more accurate ETEFs. An interesting point is that defining an objective function by the residuals of absolute acceleration spectra is susceptible to lower accuracy of $20 \%$ than when the residuals of relative acceleration spectra are considered. This highlights the importance of defining the optimal objective function in the accuracy of ETEFs.

\section{Results}

This section aims to investigate the effect of the objective function definition on the accuracy of simulated ETEFs. The accuracy of ETA20SC1X2 is compared with that of ETA20SC2X2. In the ETEF names, the number after "SC" denotes the scenario number (according to Table 1), and the number after "X" denotes the initial motion number. In fact, ETA20S1CX2 is generated by the second simulation scenario using $X_{2}$ as the initial optimization point. ETA20SC1X2 and ETA20SC2X2 are generated from a similar initial point, except different generating scenarios. Therefore, the difference between ETA20SC1X2 and ETA20SC2X2 accuracy is derived from those objective functions. The first and second scenarios are selected because the former is the current practice for generating ETEFs and the latter is the optimal simulation scenario, determined in the previous section. Targets of these ETEFs are based on FEMAP695 [21] far-field record set.

ETA20SC1X2 and ETA20SC2X2 acceleration time histories are shown in Figures 7 and 8.

ETA20SC1X2 and ETA20SC2X2 acceleration

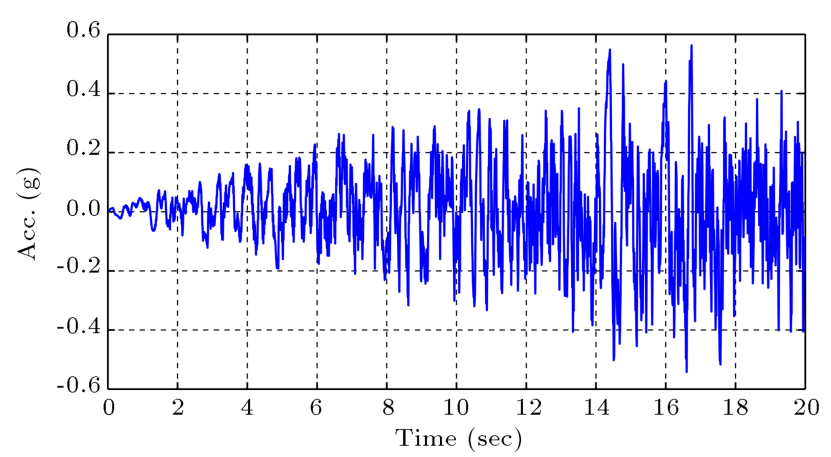

Figure 7. ETA20SC1X2 acceleration time history.

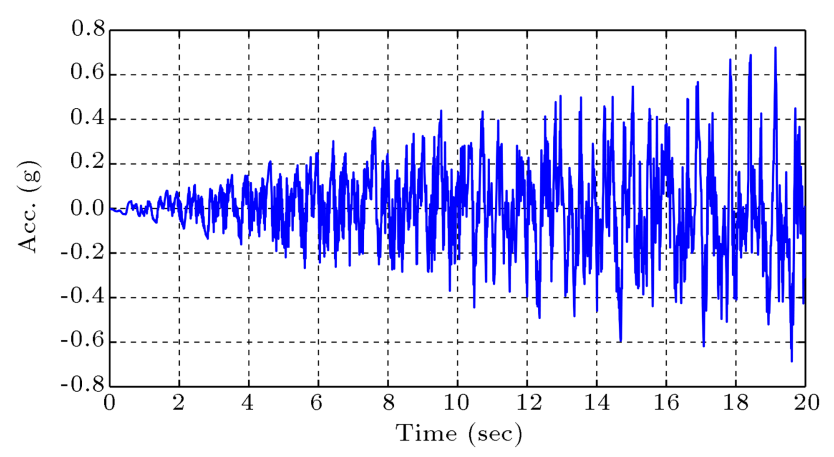

Figure 8. ETA20SC2X2 acceleration time history.

spectra are compared with targets at four time intervals, i.e., $t=5 \mathrm{sec}, 10 \mathrm{sec}, 15 \mathrm{sec}$, and $20 \mathrm{sec}$, as shown in Figure 9. It can be seen that the discrepancies between ETA20SC1X2 and targets are obviously more than those of ETA20SC2X2.

ETA20SC1X2 and ETA20SC2X2 displacement spectra are compared with targets at four time intervals, i.e., $t=5 \mathrm{sec}, 10 \mathrm{sec}, 15 \mathrm{sec}$, and $20 \mathrm{sec}$, as shown in Figure 10. It can be seen that both of these ETEFs enjoy acceptable and similar accuracy.

ETA20SC1X2 and ETA20SC2X2 velocity spectra are compared with targets at four time intervals, i.e., $t=5 \mathrm{sec}, 10 \mathrm{sec}, 15 \mathrm{sec}$, and $20 \mathrm{sec}$, as shown in 


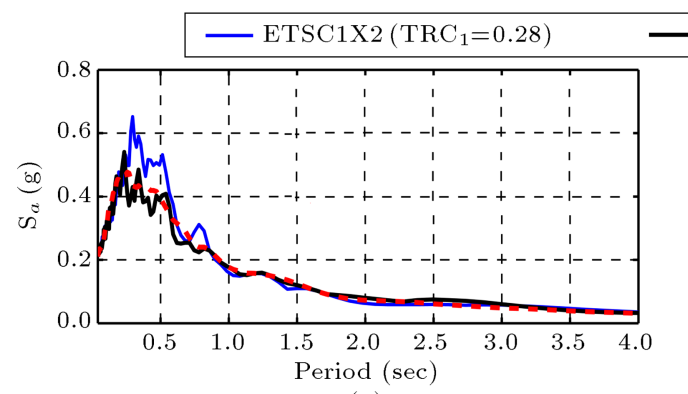

(a)

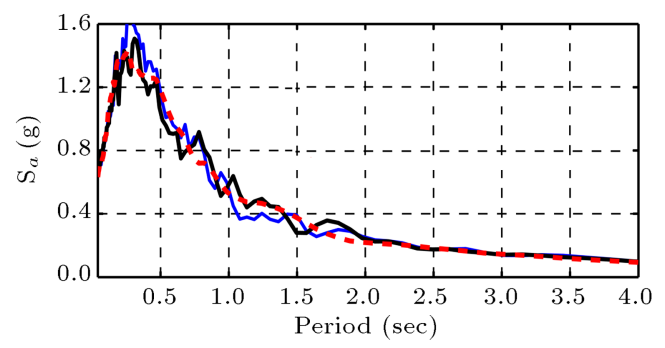

(c)

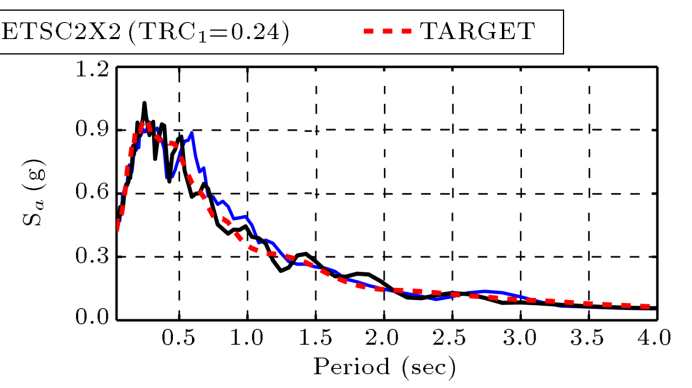

(b)

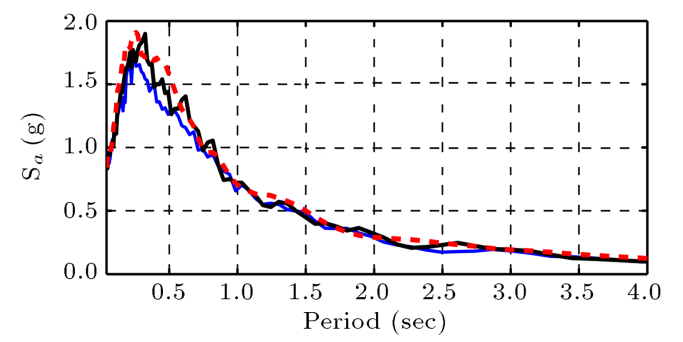

(d)

Figure 9. ETA20SC1X2 and ETA20SC $2 \mathrm{X} 2$ acceleration spectra versus targets at times: (a) $t=5 \mathrm{sec}$, (b) $t=10 \mathrm{sec},(\mathrm{c})$ $t=15 \mathrm{sec}$, and (d) $t=20 \mathrm{sec}$.

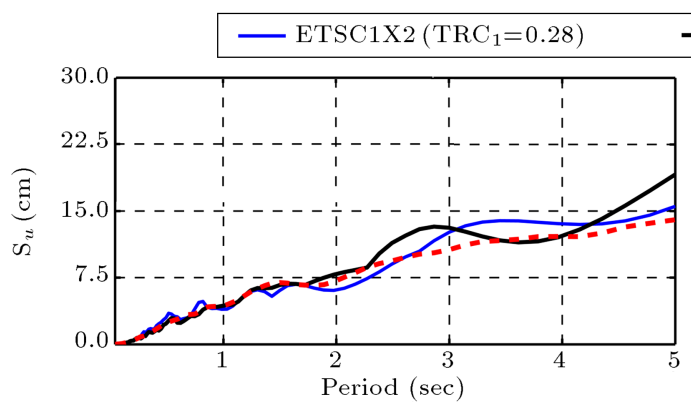

(a)

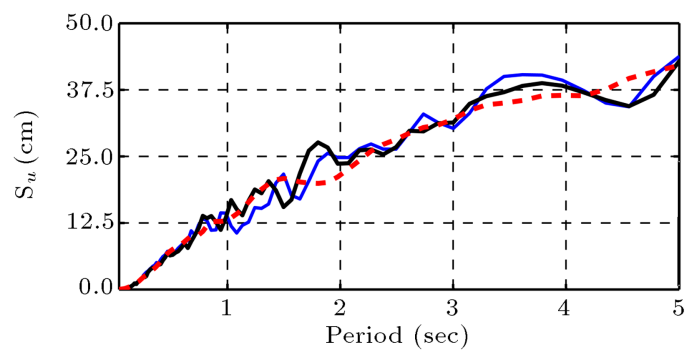

(c)

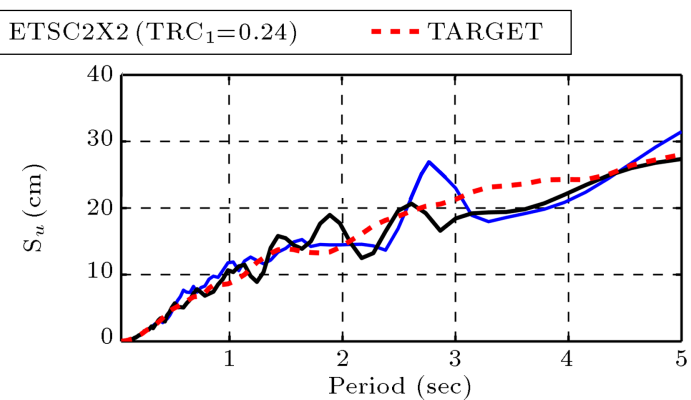

(b)

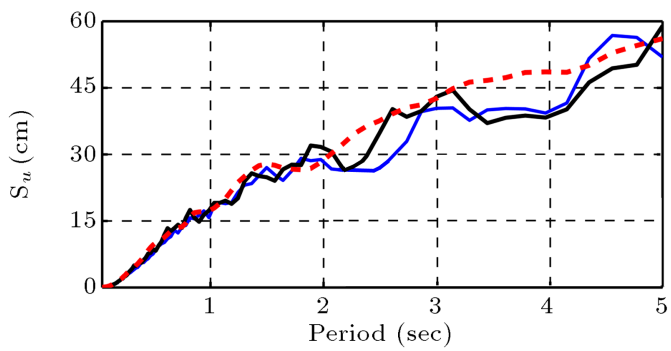

(d)

Figure 10. ETA20SC1X2 and ETA20SC2X2 displacement spectra versus targets at times: (a) $t=5$ sec, (b) $t=10$ sec, (c) $t=15 \mathrm{sec}$, and (d) $t=20 \mathrm{sec}$.

Figure 11. It can be seen that both of these ETEFs enjoy acceptable and similar accuracy.

ETA20SC1X2 and ETA20SC2X2 acceleration spectra are compared with targets at four time intervals, $T=0.06 \mathrm{sec}, 0.5 \mathrm{sec}, 1 \mathrm{sec}$, and $3 \mathrm{sec}$, as shown in Figure 12. This comparison shows that ETA20SC2X2 enjoys higher accuracy.

It can be seen that the objective function definition can appreciably influence the accuracy of simulated ETEFs. The results show higher accuracy associated with the optimal objective function than the conventional objective function definition.

\section{Conclusion}

The Endurance Time (ET) method, which is a novel time history analysis, facilitates the estimation of structural responses at different seismic intensity levels with appreciable computational time saving. The central part of the ET method is Endurance Time Excitation 


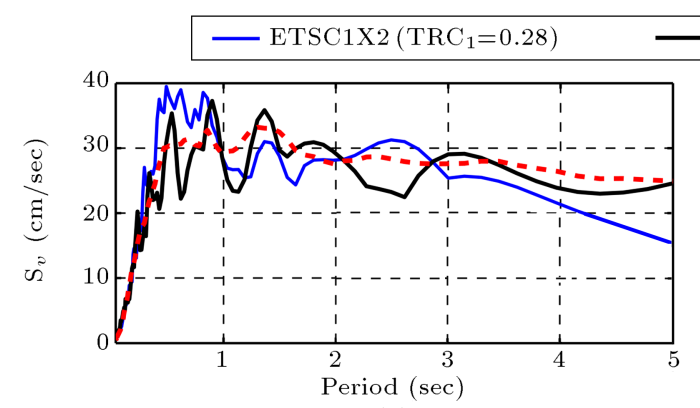

(a)

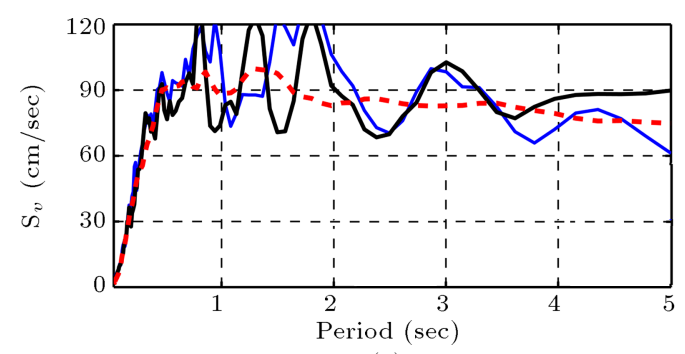

(c)

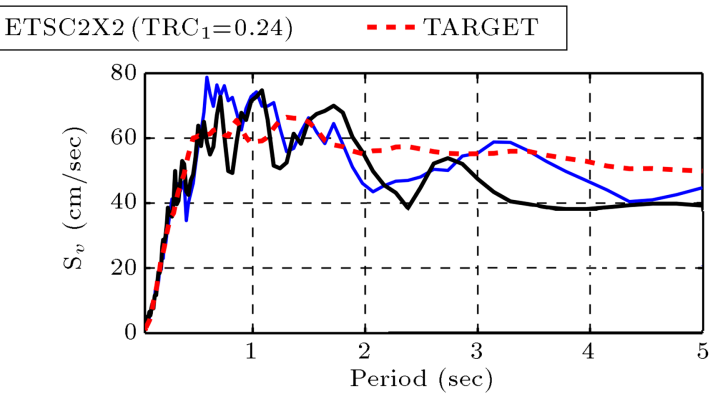

(b)

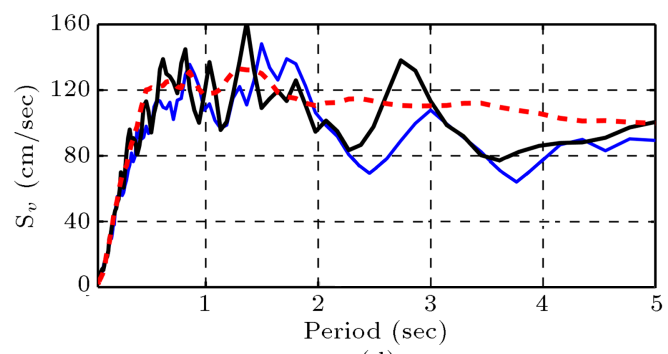

(d)

Figure 11. ETA20SC1X2 and ETA20SC2X2 velocity spectra versus targets at times: (a) $t=5 \mathrm{sec}$, (b) $t=10 \mathrm{sec}$, (c) $t=15 \mathrm{sec}$, and (d) $t=20 \mathrm{sec}$.

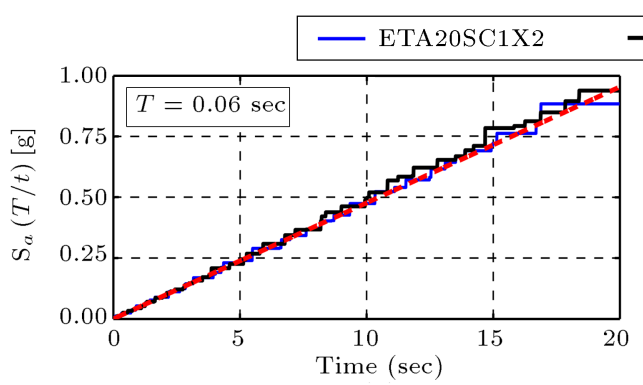

(a)

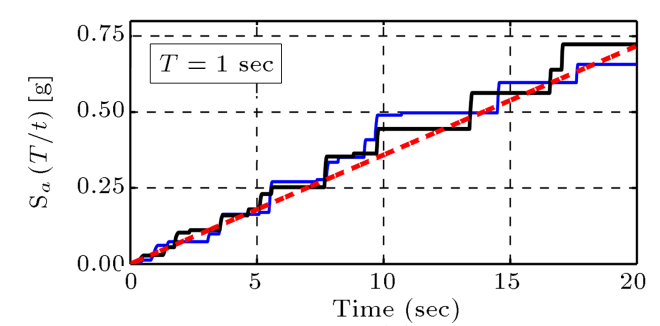

(c)

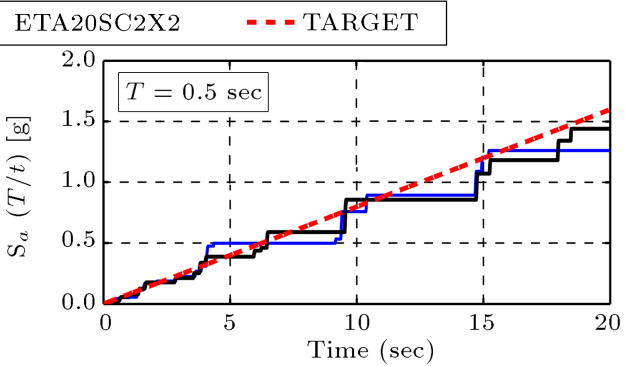

(b)

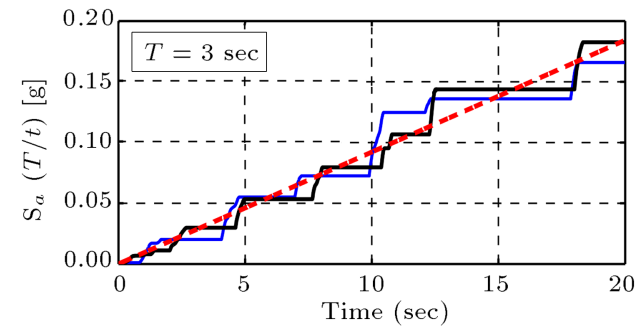

(d)

Figure 12. Comparison ETA20SC1X2 and ETA20SC2X2 acceleration spectra with targets at periods: (a) $T=0.06$ sec, (b) $T=0.5 \mathrm{sec}$, (c) $T=1 \mathrm{sec}$, and (d) $T=3 \mathrm{sec}$.

Functions (ETEFs), which are intensifying acceleration time histories imposed on structures. The concept of response spectra was used to generate ETEFs; ETEFs response spectra increase with time and must be proportional to the target response spectra at each time interval. Target response spectra could be a design code spectrum or the average response spectrum of a suite of ground motions. Unconstrained nonlinear optimization was used to solve these equations. In the context of optimization, equations must be pre- sented by objective functions. Objective functions were defined to minimize the differences between ETEFs response spectra and targets. Objective functions can be defined in several forms and have obvious effects on the results. This study presents a method to find the optimal objective function definition. In the proposed method, a criterion for ranking simulated ETEFs was introduced. Apart from introducing a criterion, the method includes ranking different ETEFs generated by different initial optimization points. In fact, the 
method removes the dependence of the results on the initial optimization points. The proposed method was applied to find the optimal simulating scenario, where acceleration, displacement, and velocity spectra can be included in the process. The results are listed below:

1. It was shown that the best simulation scenario for an initial optimization point might differ from another initial point. This issue implies that finding an optimal scenario requires considering the initial optimization point variability. Otherwise, one must determine the optimal objective function for the desired initial point, which is not practical;

2. Applicability of the proposed method for removing the effect of the initial point on determining the optimal objective function was shown. The optimal objective function can be determined by considering the initial point variability using the proposed method;

3. It was shown that the objective function, which considers the residuals of relative acceleration spectra, produced more accurate ETEFs in the linear domain of ETEFs simulation. Current practices in simulating linear ETEFs include acceleration and the residuals of absolute displacement spectra in the objective function;

4. It was found that the optimal objective function result was not dependent on the considered importance vector. Importance vector quantifies the importance of each dynamic characteristic in evaluating the accuracy of ETEFs. This can be attributed to the fact that information concerning different linear response spectra is closely related together;

5. It was made clear that the objective function definition could affect the simulated ETEFs results by $35 \%$. This effect can be attributed to the fact that the objective function definition changes the directions of finding the optimum point and, thus, the optimal definition provides more intelligent directions toward the optimum point;

6. Response spectra of the ETEF generated by the optimal objective function were compared with the results obtained from the conventional objective function definition. The results showed an improvement in the response spectra accuracy of the newly generated ETEFs. This fact highlights the importance of objective function definition in generating ETEFs.

\section{References}

1. Estekanchi, H.E., Vafai, A., and Sadeghazar, M. "Endurance time method for seismic analysis and design of structures", Scientia Iranica, 11(4), pp. 361-370 (2004).

2. Krawinkler, H. and Seneviratna, S. "Pros and cons of a pushover analysis of seismic performance evaluation", Eng. Struct., 20(4-6), pp. 452-464 (1998).

3. Federal Emergency Management Agency (FEMA), Pre-Standard and Commentary for the Seismic Rehabilitation of Buildings, FEMA-356, Washington, DC (2000).

4. Federal Emergency Management Agency (FEMA), NEHRP Guidelines for Seismic Rehabilitation of Buildings, Report FEMA-273, Washington, DC (1997).

5. Vamvatsikos, D. and Cornell, C.A. "Incremental dynamic analysis", Earthq. Eng. Struct. D, 31(3), pp. 491-514 (2002).

6. Kiani, J. and Khanmohammadi, M. "New approach for selection of real input ground motion records for incremental dynamic analysis (IDA)", J. Struct. Eng., 19, pp. 592-623 (2015).

7. Riahi, H.T., Estekanchi, H.E., and Vafai, A. "Application of endurance time method in nonlinear seismic analysis of SDOF Systems", J. App. Sci., 9(10), pp. 1817-1832 (2009).

8. Riahi, H.T., Estekanchi, H.E., and Vafai, A. "Seismic assessment of steel frames with the endurance time method", J. Construct. Steel. Res., 66(6), pp. 780-792 (2010).

9. Mirzaee, A. and Estekanchi, H.E. "Performance-based seismic retrofitting of steel frames by endurance time method", Earthquake Spectra, 31(1), pp. 383-402 (2015).

10. Rahimi, E. and Estekanchi, H.E. "Collapse assessment of steel moment frames using endurance time method", Earthq. Eng. Eng. Vib., 14(2), pp. 347-360 (2015).

11. Basim, M.C. and Estekanchi, H.E. "Application of endurance time method in performance-based optimum design of structures", Struct. Saf., 56, pp. 52-67 (2015).

12. Tafakori, E., Pourzeynali, S., and Estekanchi, H.E. "Probabilistic seismic loss estimation via endurance time method", Earthq. Eng. Eng. Vib., 16(1), pp. 233245 (2017).

13. Chiniforush, A.A., Estekanchi, H., and Dolatshahi, K.M. "Application of endurance time analysis in seismic evaluation of an unreinforced masonry monument", J. Struct. Eng., 23(3), pp. 827-841 (2016).

14. Vaezi, D., Estekanchi, H.E., and Vafai, A. "A parametric study of seismic response in anchored steel tanks with endurance time method", Scientia Iranica, 21(5), pp. 1608-1619 (2014).

15. Nozari, A. and Estekanchi, H.E. "Optimization of endurance time acceleration functions for seismic assessment of structures", Int. J. Optim. Civ. Eng., 2, pp. $257-277$ (2011). 
16. Kaveh, A. and Mahdavi, V.R. "Generation of endurance time acceleration functions using the wavelet transform", Int. J. Optim. Civ. Eng., 2(2), pp. 203219 (2012).

17. Kaveh, A., Kalateh, M., and Estekanchi, H.E. "Production of endurance time excitation function: The CMA evolution strategy approach", Iranian Journal of Science and Technology, Transaction of Civil Engineering, 37, pp. 383-394 (2013).

18. Mashayekhi, M. and Estekanchi, H.E. "Investigation of strong-motion duration consistency in endurance time excitation functions", Scientia Iranica, 20(4), pp. 1085-1093 (2013).

19. Mashayekhi, M. and Estekanchi, H.E. "Investigation of non-linear cycles' properties in structures subjected to endurance time excitation functions", Int. J. Optim. Civ. Eng., 3(2), pp. 239-257 (2013).

20. Mashayekhi, M. and Estekanchi, H.E. "Significance of effective number of cycle in endurance time", Asian Journal of Civil Engineering (Building and Housing), 13(5), pp. 647-657 (2012).

21. Federal Emergency Management Agency (FEMA), Quantification of Building Seismic Performance Factors, FEMA P-695, Washington, D.C (2009).

22. Moré, J.J. and Sorensen, D.C. "Computing a trust region step", SIAM. J. Sci. Stat. Comput., 3, pp. 553572 (1983).

23. Mashayekhi, M., Estekanchi, H.E., and Vafai, H. "Simulation of endurance time excitations using increasing sine functions", Int. J. Optim. Civ. Eng., 9(1), pp. 6577 (2018).

\section{Biographies}

Mohammadreza Mashayekhi is a graduate of Khaje Nasir Toosi University (KNTU). He received his MSc degree from Sharif University of Technology (SUT), Tehran, Iran. He pursued his studies at PhD level in SUT since 2013. His research interests include structural engineering, earthquake engineering, optimization, and performance-based design.

Homayoon Estekanchi is a Professor of Civil Engineering at Sharif University of Technology (SUT), Tehran, Iran. He received his $\mathrm{PhD}$ in Civil Engineering from SUT in 1997; since then, he has been a faculty member of the university. He is a member of the Iranian Construction Engineers Organization, ASCE, Iranian Inventors Association, and several other professional associations. His research interests include a broad area of topics in structural and earthquake engineering with particular focus on the design of tall buildings and industrial structures.

Hasan Vafai is a Professor of Civil Engineering at Sharif University of Technology. He has authored/coauthored numerous papers in different fields of engineering: applied mechanics, biomechanics, and structural engineering (steel, concrete, timber, and offshore structures). He has also been active in the area of higher education and has delivered lectures and published papers on challenges of higher education, the future of science and technology, and human resources development. 